Funding

No external funding received.

Conflict of interest

None.
Nils Dennhardt, Christiane Beck, Dirk Huber \& Robert Sümpelmann Clinic for Anesthesiology, Hanover Medical School, Hanover, Germany

Email:Dennhardt.Nils@mh-hannover.de

doi:10.1111/pan.13079

2 Sümpelmann R, Becke K, Brenner S et al. Perioperative intravenous fluid therapy in children: guidelines from the Association of the Scientific Medical Societies in Germany. Paediatr Anaesth 2016. doi:19.1111/pan. 13007

3 Rhondali O, Juhel S, Mathews S et al. Impact of sevoflurane anesthesia on brain oxygenation in children younger than 2 years Pediatr Anesth 2014; 24: 734-740.

4 Dennhardt N, Beck C, Huber D et al. Impact of preoperative fasting times on blood glucose concentration, ketone bodies and acid-base balance in children younger than 36 months: a prospective observational study. Eur J Anaesthesiol 2015; 32: 1-5.

\title{
Reply to Nielsen, Dominic; Visram, Anil, regarding their comment 'Comment on Tait AR, Bickham R, O'Brien LM, Quinlan M, Voepel-Lewis T. The STBUR questionnaire for identifying children at risk for sleep-disordered breathing and postoperative opioid-related adverse events - potential confounders'
}

SIR-We thank Drs. Nielsen and Visram for their interest in our paper regarding the use of the STBUR tool to identify children at risk for sleep-disordered breathing and postoperative opioid-related adverse events (1). Given that postoperative opioid-related adverse events were our primary outcome measure, we recruited children undergoing procedures requiring postoperative opioids. To this end, the majority of children in the study were those who had undergone adenotonsillectomy ( $\mathrm{T} \& \mathrm{~A}$ ) and by association, many who also had sleep-disordered breathing. We agree with the authors that many children undergoing $\mathrm{T} \&$ A surgery are at increased risk for perioperative respiratory adverse events but our point here was to determine if the STBUR tool could identify those children (undergoing both airway and nonairway procedures) who might be at greater risk because of their sleepdisordered breathing symptoms. In this study, we showed that for all procedures children who presented with $\geq 3$ STBUR symptoms had a twofold increase in the risk of perioperative respiratory adverse events compared with children with $<3$ symptoms. We also noted at the time that the incidence of perioperative respiratory adverse events among children with sleepdisordered breathing was lower than in our initial evaluation of the STBUR (2) tool and speculated that this may have been due to an increased awareness of sleep-disordered breathing as a risk factor in our institution and implementation of risk mitigation strategies. Drs. Nielsen and Visram correctly point out, however, that there was no statistical difference in perioperative respiratory adverse events between children with and without sleep-disordered breathing who underwent $\mathrm{T} \& \mathrm{~A}$ surgery in the current study. In response to this comment, we re-examined the data from our previous study (2) (of which $85 \%$ were non$\mathrm{T} \& \mathrm{~A}$ surgeries) and found weak evidence to suggest that children with sleep-disordered breathing (per STBUR) undergoing $\mathrm{T} \& \mathrm{~A}$ procedures had a greater than twofold increase in the risk of perioperative respiratory adverse events (OR, 95\% CI $=2.56,0.74$ 9.06). For children undergoing non-T \& A surgery with sleep-disordered breathing, there was a greater than fourfold increased risk of perioperative respiratory adverse events (OR, 95\% CI $=4.43,1.08-21.0$ ). Again, we can only speculate on the reasons for 
weaker evidence in the $\mathrm{T} \& \mathrm{~A}$ group but suggest that in both studies we were likely under-powered. In the present study for example, the sample size was based on the ability to detect differences in postoperative oxygen desaturation of $<90 \%$.

With respect to the authors' comment regarding age as a potential confounder, we completely agree that younger children are typically at increased risk for perioperative respiratory adverse events and this indeed was confirmed in our study. For example, although we did not present the data in the paper, younger children (2-7 years) who underwent both $\mathrm{T} \& \mathrm{~A}$ and non- $\mathrm{T} \&$ A surgery had significantly greater risk of perioperative respiratory adverse events compared with children 817 years. Furthermore, the risk among younger children undergoing $\mathrm{T} \& \mathrm{~A}$ was, as expected, greater than similarly aged children who underwent non-T \& A surgery $(\mathrm{OR}, 95 \% \mathrm{CI}=3.73,2.29-6.08)$. In the paper, we showed that the ability of the STBUR to identify children at increased risk for perioperative respiratory adverse events was consistent for both young and older children. However, if, as the authors suggest, we stratify these age data by type of surgery, we find that there was only very weak evidence toward a greater incidence of perioperative respiratory adverse events in the sleep-disordered breathing group compared with the no-sleep-disordered breathing group. The only poor level of evidence may be due to sample size and/or as speculated above, perhaps as a result of optimized management strategies (i.e., young children undergoing non- $\mathrm{T} \& \mathrm{~A}$ : OR, $95 \% \mathrm{CI}=6.50,0.441-187.7)$ and young children undergoing $\mathrm{T} \& \mathrm{~A}$ : OR, 95\% $\mathrm{CI}=1.41,0.81-2.44)$. When analyzing the same data for older children, there was no evidence for a difference between children with and without SDB.

The goals of this study were to confirm our previous data regarding the ability of the STBUR to identify children at risk for perioperative respiratory adverse events for a variety of surgical procedures but more specifically to examine if STBUR could be used to identify children at risk for postoperative opioid-related adverse events. While Drs. Nielsen and Visram raise some valid questions regarding the potential confounding effects of age and $\mathrm{T} \& \mathrm{~A}$, the observation that the STBUR is able to identify 'at risk' children in the absence of these confounders suggests its utility as a simple and practical preoperative risk assessment tool.

\section{Conflicts of interests}

No conflicts of interest declared.

\section{Funding}

None.

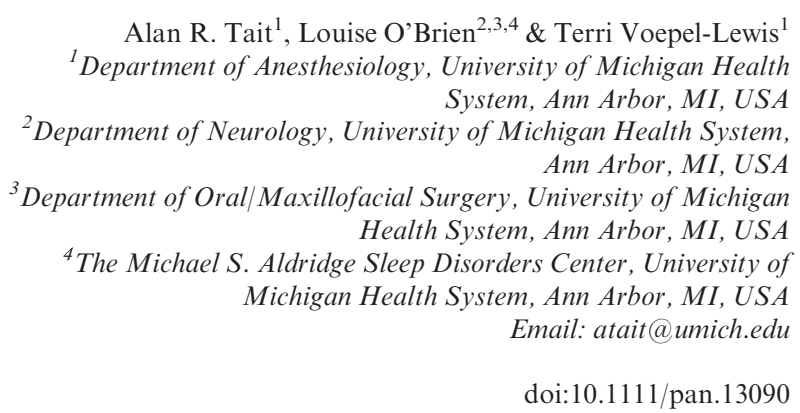

\section{References}

1 Tait AR, Bickham R, O'Brien L et al. The STBUR questionnaire for identifying children at risk for sleep-disordered breathing and postoperative opioid-related adverse
2 Tait AR, Voepel-Lewis T, Christensen R et al. The STBUR questionnaire for predicting perioperative respiratory adverse events in children at risk for sleep-disordered breathing. Pediatr Anesth 2013; 23: 510-516.

\section{Airway management in a neonate with tracheoesophageal fistula and subglottic stenosis}

SIR - A three-day-old neonate with a tracheoesophageal fistula (TEF) was scheduled for thoracotomy and fistula ligation. Following delivery, he had respiratory distress for which intubation was attempted, which was unsuccessful. A CT scan showed esophageal atresia and carinal
TEF with subglottic stenosis (Figure 1). The baby was transferred to our tertiary hospital and administered nasal continuous positive airway pressure preoperatively.

In view of previous failed attempts at intubation and CT images, surgeons were kept on stand-by for 